\title{
Avaliação da aceitação de Tecnologias Educacionais Digitais aplicadas ao ensino-aprendizagem de matemática
}

\author{
Gibran Medeiros Chaves de Vasconcelos, UNIVASF, gibran.vasconcelos@ifsertao-pe.edu.br, \\ https://orcid.org/0000-0002-0836-0428 \\ João Carlos Sedraz Silva, UNIVASF, joao.sedraz@univasf.edu.br, \\ https://orcid.org/0000-0002-4082-9652 \\ Luciano Sedraz Silva, SEC, lucianosedraz@yahoo.com.br, \\ https://orcid.org/0000-0002-3347-374X
}

\begin{abstract}
Resumo. Este artigo teve como objetivo avaliar a aceitação dos professores em relação à utilização das Tecnologias Educacionais Digitais (TED) adotadas para o ensinoaprendizagem de Matemática. Para isso, foi realizada uma pesquisa de opinião com os professores de matemática do Instituto Federal de Educação, Ciência e Tecnologia do Sertão Pernambucano (IFSertãoPE). O método utilizado fundamentou-se no Modelo de Aceitação Tecnológica (TAM, do inglês Technological Acceptance Model). Para avaliação da aceitação dos professores, foi elaborado um questionário contendo declarações para cada um dos construtos do TAM: facilidade de uso percebida, utilidade percebida, intenção de uso e variáveis externas. Os resultados indicaram uma boa aceitação dos participantes da pesquisa em relação às TED avaliadas. No entanto, apontam que há questões que precisam ser enfrentadas pela instituição de ensino, a fim de que as TED possam auxiliar no processo de ensino-aprendizagem de matemática.
\end{abstract}

Palavras-chave: Educação, Formação de Professores, Utilização de Tecnologia.

\section{Assessing the acceptance of Digital Educational Technologies, teaching-learning mathematics}

\begin{abstract}
This article aimed to evaluate the acceptance of teachers in relation to the use of Digital Educational Technologies (TED), adopted for the teaching learning of Mathematics. For this, an opinion poll was fulfilled with the math teachers of the Federal Institute of Education, Science and Technology of the Sertão Pernambucano. The method applied is based on the Technological Acceptance Model (TAM). To evaluation the acceptance of teachers, a questionnaire was prepared containing statements for each of the constructs: perceived ease of use, perceived utility, intention to use and external variables. The results indicated a good acceptance of the research participants in relation to the evaluated TED. However, they point out that there are issues that need to be faced by the educational institution, so that TED can assist in the mathematics teaching-learning process.
\end{abstract}

Keywords: Education, Teacher Training, Technology Utilization.

\section{Introdução}

As Tecnologias Educacionais Digitais (TED) estão cada vez mais presentes nas instituições de ensino, tornando-se uma ferramenta relevante para o ensino-aprendizagem dos educandos. Para Retzlaff et al. (2018, p.2), "Ferramentas computacionais agregadas ao uso de metodologias conduzem a utilização adequada de recursos, tornando-se aliadas do professor no processo de ensino e aprendizagem".

Por meio da tecnologia, é possível obter uma maior interação do educando, permitindo-lhe sair de uma postura passiva e assumir uma participação ativa na construção do seu conhecimento. Nesse sentido, Grinspun et al. (2016, p. 66) afirmam: "As novas tecnologias interativas permitem a maior participação, a intervenção, à multiplicidade de 
conexões. Ao mesmo tempo o jovem pode ser ator e autor de sua criatividade, seu discurso, sua ação, sua representação".

Contudo, geralmente, as TED podem ser usadas de maneira proativa em benefício ao ensino de Matemática, sendo possível tornar as aulas mais dinâmicas e atrativas para um público que já está acostumado com a interatividade e a mobilidade.

Lopes et al. (2016, p. 4) destacam: "O desafio maior está em fazer com que se faça uso desses recursos como facilitador da aprendizagem, pois tanto professores como alunos, costumeiramente, usam esses recursos somente para jogos, redes sociais e entretenimento sem fins educativos". Por isso, o uso desses artefatos tecnológicos constitui uma prática pedagógica importante, que não deve abranger apenas os docentes, mas gestores educacionais, discentes e coordenação pedagógica.

De acordo com Da Silva et al. (2018, p. 61): “A inserção de tecnologias no meio educacional não é importante se não houver, em paralelo, a capacitação do atual corpo docente que, muitas vezes, opta por não utilizar tais recursos em função de não possuir o conhecimento necessário para a utilização destes".

O empenho em envolver os profissionais da educação gera o despertar de um pensamento crítico, ao propor-lhes a análise constante e de forma simultânea da utilização das TED com a melhoria da aprendizagem do educando.

Basso e Notare (2015, p.4) discorrem acerca das ideias anteriores ao afirmarem que:

É importante, no momento de pensar em atividades com o uso de tecnologias para a sala de aula, ter claro os objetivos que queremos alcançar e escolher a tecnologia de modo a atendê-los, ao invés de, simplesmente, utilizar a tecnologia para tornar a aula mais atraente, mas de forma tangente e superficial, ou até mesmo prejudicial.

Nessa perspectiva, pode-se questionar: Qual a aceitação dos professores em relação à utilização das TED adotadas para o ensino-aprendizagem de Matemática? Diante dessa questão, o objetivo geral desta pesquisa foi avaliar a aceitação dos professores em relação à utilização das TED adotadas para o ensino-aprendizagem de Matemática.

A investigação foi realizada em uma Instituição Federal de Educação, localizada no Semiárido do Estado de Pernambuco. Para alcançar o objetivo proposto, foi conduzida uma pesquisa de opinião baseada no Modelo de Aceitação de Tecnologia (TAM, do inglês Technology Acceptance Model), que será explicado na seção 3.

Este artigo está organizado em cinco seções, incluindo esta introdução. Na segunda seção, são apresentadas as Tecnologias Educacionais Digitais adotadas para o ensinoaprendizagem de matemática em instituições de Ensino Médio no Brasil; na terceira seção, o método utilizado para análise de aceitação das TED; na quarta seção, a discussão dos resultados; na quinta seção, as considerações finais da pesquisa.

\section{Tecnologias Educacionais Digitais adotadas para o ensino-aprendizagem de matemática}

Esta seção destina-se a apresentar as TED, com base em um mapeamento sistemático (De Vasconcelos et al., 2020, p.2) realizado pelos autores deste trabalho, buscando "[...] identificar características relevantes de projetos apoiados por TED adotados para o ensinoaprendizagem de Matemática em instituições de Ensino Médio no Brasil”.

No mapeamento sistemático (De Vasconcelos et al., 2020, p.2), após os critérios de inclusão e exclusão, observou- se o emprego de doze (12) tecnologias, distribuídas nos trinta e nove (39) artigos científicos selecionados. Esses artigos foram identificados de acordo com a ordem alfabética de suas respectivas referências. A estratégia de busca, o protocolo de condução e os resultados obtidos em cada uma das etapas podem ser visualizados no endereço:( https://bit.ly/3bEFNJH).

O Quadro 1 mostra as TED utilizadas, o identificador do artigo em que foi empregada a tecnologia e o número de artigos relacionados para cada tecnologia. 
Quadro 1. TED utilizadas.

\begin{tabular}{l|c|c}
\hline \multicolumn{1}{c|}{ Tecnologia Educacional Digital } & Identificador do artigo & Número de artigos relacionados \\
\hline Calculadora & $7 ; 26$ & 2 \\
\hline Chatbot & 27 & 6 \\
\hline Fórum de discussão & $10 ; 11 ; 12 ; 26 ; 29 ; 31$ & 3 \\
\hline Jogos educativos & $4 ; 16 ; 36$ & 6 \\
\hline Planilha eletrônica & $1 ; 6 ; 21 ; 29 ; 33 ; 39$ & 2 \\
\hline Questionários online & $3 ; 26$ & 3 \\
\hline Serviço de busca na internet & $7 ; 23 ; 26$ & 3 \\
\hline Simuladores & $8 ; 23 ; 36$ & 5 \\
\hline Sistema de gestão de aprendizagem & $11 ; 12 ; 26 ; 27 ; 28$ & 2 \\
\hline Sistema de tutor inteligente & $30 ; 31$ & 22 \\
\hline Software de geometria dinâmica & $2 ; 5 ; 9 ; 11 ; 12 ; 13 ; 14 ; 15 ; 17 ; 18 ; 19 ; 22 ; 24 ;$ & 11 \\
\hline Vídeos sob demanda & $25 ; 28 ; 31 ; 32 ; 34 ; 35 ; 37 ; 38 ; 39$ & $2 ; 12 ; 14 ; 20 ; 27 ; 29 ; 31 ; 33 ; 34$ \\
\hline
\end{tabular}

Fonte: De Vasconcelos et al. (2020, p. 6).

As TED identificadas no referido mapeamento nortearam o método adotado nesta pesquisa, que está detalhado na seção 3 .

\section{Método}

Para Prodanov e Freitas (2013, p. 24), “[...] método científico é um conjunto de procedimentos adotados com o propósito de atingir o conhecimento". Esta pesquisa é fundamentada no Modelo de Aceitação de Tecnologia (TAM) e teve como referência os estudos de Davis et al. (1989), Dias et al. (2011) e Persico et al. (2014).

O TAM foi proposto por Davis et al. (1989) para avaliar, de acordo com a percepção dos usuários, os motivos que os levam a aceitar ou rejeitar uma tecnologia. O núcleo do TAM está fundamentado em dois construtos: facilidade de uso percebida e utilidade percebida. $\mathrm{O}$ primeiro construto refere-se ao grau em que o usuário acredita que a adoção de uma determinada tecnologia seria livre de esforço, enquanto o segundo construto diz respeito ao grau em que uma pessoa acredita que a utilização de um determinado sistema melhoraria o seu desempenho no trabalho (PERSICO et al., 2014).

O construto intenção de uso está relacionado aos dois anteriores (facilidade de uso percebida e utilidade percebida). Dias et al. (2011, p.135) explicam que a intenção de uso "[...] seria determinada em conjunto pela atitude de uso do indivíduo com relação ao uso real do sistema e pela utilidade percebida, cada uma exercendo um peso relativo".

Esta pesquisa caracteriza-se por ser do tipo aplicada (quanto à sua natureza), descritiva (quanto ao seu objetivo) e quali-quantitativa (quanto à sua forma de abordagem).

\subsection{Participantes}

A pesquisa foi desenvolvida nos sete Campi do Instituto Federal de Educação, Ciência e Tecnologia do Sertão Pernambucano (IFSertãoPE) localizado no Semiárido do Estado de Pernambuco, e dela participaram 17 (dezessete) professores de matemática que lecionaram nos cursos técnicos integrados ao nível médio.

A maioria dos participantes tinha idade igual ou inferior a 39 anos (76,5\%), sendo em sua totalidade do sexo masculino. Embora haja três (3) professoras de matemática na Instituição, elas optaram por não participar. Os sujeitos da pesquisa foram distribuídos da seguinte forma: três (3) estavam lotados no Campus Floresta, dois (2) no Campus Ouricuri, cinco (5) no Campus Petrolina, um (1) no Campus Petrolina Zona Rural um (1) no Campus Salgueiro, dois (2) no Campus Santa Maria da Boa Vista e três (3) estavam lotados no Campus Serra Talhada (Quadro 2).

Quando questionados sobre a formação acadêmica e o tempo que lecionam matemática (Quadro 2), verificou-se que 70,6\% dos professores possuíam o título de mestre e $29,4 \%$ o de especialista. Já em relação ao tempo que ensinam matemática, constatou-se que maioria dos participantes da pesquisa (64,7\%) ministra a disciplina entre 10 e 20 anos.

Após a coleta inicial dos dados, a pesquisa foi direcionada a investigar o conhecimento, a habilidade e a utilização das TED pelos professores participantes. De acordo V. $19 \mathrm{~N}^{\circ} 1$, julho, 2021 RENOTE 
com o Quadro 2, percebe-se que 52,9\% dos professores não passaram por formação com foco em TED. Quanto ao nível de habilidade com o uso de equipamentos (computadores, tablets ou smartphones) e software em geral, 70,5\% dos professores afirmaram possuir um nível de conhecimento bom ou ótimo, enquanto $29,4 \%$ consideram razoável.

Quadro 2. TED utilizadas.

\begin{tabular}{|c|c|c|c|c|c|c|c|}
\hline & & & & \multirow{2}{*}{\multicolumn{2}{|c|}{\begin{tabular}{c|r} 
Questão & Alternativa \\
\end{tabular}}} & \multirow{2}{*}{\multicolumn{2}{|c|}{$\mathrm{N}^{*}$}} \\
\hline Questão & Alternativa & $\mathbf{N}^{*}$ & $\%$ & & & & \\
\hline \multirow{4}{*}{ Qual a sua idade? } & Menos de 30 anos & 2 & $11,8 \%$ & \multirow{9}{*}{$\begin{array}{l}\text { Qual o seu nivel de habilidade com } \\
\text { o uso de equipamentos } \\
\text { (computadores, tablets ou } \\
\text { smartphones) e softwares em } \\
\text { geral? }\end{array}$} & Muito ruim & 0 & $0 \%$ \\
\hline & Entre 30 e 39 anos & 11 & $64,7 \%$ & & & & \\
\hline & Entre 40 e 49 anos & 4 & $23,5 \%$ & & Ruim & 0 & $0 \%$ \\
\hline & Acima de 50 anos & 0 & $0 \%$ & & Razoável & 5 & $29.4 \%$ \\
\hline \multirow{2}{*}{ Qual o seu sexo? } & Feminino & 0 & $0 \%$ & & Razodvel & & \\
\hline & Masculino & 17 & $100 \%$ & & Boa & 9 & $52,9 \%$ \\
\hline \multirow{8}{*}{ Qual o seu Campus de lotaçāo? } & Campus Floresta & 3 & $17,6 \%$ & & & & \\
\hline & Campus Ouricuri & 2 & $11,8 \%$ & & Ótima & 3 & $17,6 \%$ \\
\hline & Campus Petrolina & 5 & $29,4 \%$ & & & & \\
\hline & Campus Petrolina Zona Rural & 1 & $5,9 \%$ & \multirow{13}{*}{$\begin{array}{l}\text { Qual (is) TED você já utilizou em } \\
\text { sala de aula? }\end{array}$} & \begin{tabular}{|l} 
Calculadora \\
\end{tabular} & 0 & $0 \%$ \\
\hline & Campus Salgueiro & 1 & $5,9 \%$ & & Chatbot & 0 & $0 \%$ \\
\hline & Campus Santa Maria da Boa Vista & 2 & $11.8 \%$ & & \begin{tabular}{|l|} 
Fórum de discussão \\
\end{tabular} & 11 & $64,7 \%$ \\
\hline & Campus Serra Talhada & 3 & $17,6 \%$ & & \begin{tabular}{|l} 
Jogos educativos \\
\end{tabular} & 0 & $0 \%$ \\
\hline & Especializacạ̃o & 5 & $\frac{11,0 \%}{29,4 \%}$ & & \begin{tabular}{|l|} 
Planilha eletrônica \\
\end{tabular} & 8 & $47,1 \%$ \\
\hline Qual a sua maior titulação & Mestrado & 12 & $70,6 \%$ & & Questionários online & 0 & $0 \%$ \\
\hline \multirow{4}{*}{$\begin{array}{l}\text { Há quanto tempo você leciona } \\
\text { matemática }\end{array}$} & Menos de 5 anos & 2 & $11,8 \%$ & & \begin{tabular}{|l} 
Servico de busca na internet \\
Simuladores
\end{tabular} & 0 & $0 \%$ \\
\hline & Entre 5 e 9 anos & 2 & $11,8 \%$ & & \begin{tabular}{|l|} 
Simuladores \\
Sistema de gestão de aprendizagem
\end{tabular} & $\begin{array}{c}0 \\
15\end{array}$ & $\begin{array}{l}0 \% \\
88,2 \%\end{array}$ \\
\hline & Entre 10 e 20 anos & 11 & $64,7 \%$ & & \begin{tabular}{|l} 
Sistema de tutor inteligente \\
\end{tabular} & 0 & $0 \%$ \\
\hline & Acima de 20 anos & 2 & $11,8 \%$ & & Soffuare de geometria dinâmica & 12 & $70,6 \%$ \\
\hline \multirow{3}{*}{$\begin{array}{l}\text { Você cursou algum curso/formaçẫo com } \\
\text { foco em Tecnologias Educacionais } \\
\text { Digitais (TED)? }\end{array}$} & Sim & 8 & $47,1 \%$ & & \begin{tabular}{|l} 
Videos sob demanda \\
\end{tabular} & 17 & $100 \%$ \\
\hline & Não & 9 & $52,9 \%$ & & Nenhuma & 0 & $0 \%$ \\
\hline & & & & & Outro (a): & 0 & $0 \%$ \\
\hline
\end{tabular}

Fonte: Dados da pesquisa.

Os professores foram questionados sobre quais TED já utilizaram em sala de aula (Quadro 2). Constatou-se a utilização de vídeos por todos os respondentes (100\%), seguidos de Sistema de gestão de aprendizagem $(88,2 \%)$, Software de geometria dinâmica $(70,6 \%)$, Fórum de discussão $(64,7 \%)$, Planilha eletrônica $(47,1 \%)$. Verificou-se, ainda, que não houve o emprego de calculadora, chatbot, jogos educativos, questionários online, serviço de busca na internet, simuladores e sistema de tutor inteligente nas práticas de ensino dos participantes da pesquisa.

\subsection{Procedimentos}

A coleta de informações foi realizada entre os dias 18 de agosto e 16 de setembro de 2020, por meio de questionários online elaborados no Google formulários ${ }^{1}$.

Para avaliação da aceitação dos professores sobre a utilização das TED no ensinoaprendizagem de matemática, o questionário contou com declarações relacionadas a cada um dos construtos do TAM (Quadro 3). As declarações foram associadas ao uso de uma escala psicométrica de Likert, que variava de 1 a 5 , sendo: $1=$ Discordo completamente e $5=$ Concordo plenamente.

De acordo com Vieira (2009, p. 42), "O escalonamento das opções de respostas foi proposto por Likert, que também sugeriu que os questionários fossem formados não apenas por questões, mas por declarações [...]". Em relação a estas, Vieira $(2009$, p.42) explica que "o uso de declarações - e não apenas de questões - aumenta a flexibilidade do questionário e pode tornar a tarefa do participante mais agradável".

Os valores do índice Alfa de Cronbach $(\alpha)$ obtidos com base nas declarações associadas a cada construto são apresentados no Quadro 4. Esse índice é utilizado para avaliar a consistência interna do instrumento de coleta de dados. Segundo Hair et al. (2009), $\alpha$ não deve atingir valores inferiores a 0,6 .

No questionário também foram incluídas questões abertas (Quadro 5), para verificar os pontos positivos e negativos quanto ao emprego das TED. Sob a perspectiva do TAM, esses pontos podem ser compreendidos como variáveis externas, que podem influenciar na aceitação e que devem ser consideradas para uma adoção adequada das tecnologias. Por fim, uma questão para um comentário geral sobre as TED.

\footnotetext{
${ }^{1}$ https://www.google.com/intl/pt-BR/forms/about/
} 
Quadro 3. Declarações associadas ao Modelo de Aceitação de Tecnologia.

Itens com escala de Likert de cinco (5) pontos

\begin{tabular}{|c|c|c|}
\hline \multicolumn{3}{|c|}{$\begin{array}{c}\text { Itens com escala de Likert de cinco (5) pontos } \\
\text { (1- discordo completamente, } 2 \text { - discordo, } 3 \text { - não sei ou indiferente, } 4 \text { - concordo, } 5 \text { - concordo completamente) }\end{array}$} \\
\hline Construto & Declaração & Descrição \\
\hline \multirow{4}{*}{$\begin{array}{l}\text { Facilidade } \\
\text { percebida }\end{array}$} & D01 & As tecnologias são fáceis de serem manipuladas \\
\hline & $\mathrm{D} 02$ & Estou acostumado a elaborar aulas com o emprego de tecnologias \\
\hline & D03 & Consigo utilizar as tecnologias sem a necessidade de um curso de formação \\
\hline & D04 & O uso das tecnologias facilita a minha explicação dos conteúdos abordados em sala de aula \\
\hline \multirow{4}{*}{$\begin{array}{l}\text { Utilidade } \\
\text { percebida }\end{array}$} & D05 & Proporcionam aulas mais dinâmicas e atrativas \\
\hline & D06 & Motivam os alunos a serem mais participativos nas aulas \\
\hline & D07 & $\begin{array}{l}\text { O uso das tecnologias permite uma maior visualização e compreensão dos conteúdos } \\
\text { ministrados }\end{array}$ \\
\hline & D08 & Auxiliam no ensino-aprendizagem de matemática \\
\hline \multirow{4}{*}{$\begin{array}{l}\text { Intenção de } \\
\text { Uso }\end{array}$} & D09 & $\begin{array}{l}\text { Gostaria de ter cursado disciplinas com foco em tecnologias durante a minha formação } \\
\text { acadêmica }\end{array}$ \\
\hline & D10 & Recomendo o emprego de tecnologias em sala de aula \\
\hline & D11 & $\begin{array}{l}\text { Gostaria de realizar capacitação em tecnologias apoiadas ao ensino-aprendizagem de } \\
\text { matemática }\end{array}$ \\
\hline & D12 & Estou motivado(a) a utilizar as tecnologias nas disciplinas que leciono \\
\hline
\end{tabular}

Fonte: Elaborado pelos autores (2020).

Quadro 4. Alfa de Cronbach das declarações fundamentadas no TAM.

\begin{tabular}{c|c}
\hline Construto & $\alpha$ \\
\hline Facilidade percebida & 0,74 \\
\hline Utilidade percebida & 0,89 \\
\hline Intenção de Uso & 0,79 \\
\hline
\end{tabular}

Fonte: Elaborado pelos autores (2020).

Quadro 5. Questões abertas para a identificação de variáveis externas.

Questões abertas relacionadas ao uso das TED como ferramenta pedagógica

Quais os pontos positivos quanto ao emprego das TED: fórum de discussão, planilha eletrônica, sistema de gestão de aprendizagem, software de geometria dinâmica, vídeo sob demanda?

Quais os pontos negativos quanto ao emprego das TED: fórum de discussão, planilha eletrônica, sistema de gestão de aprendizagem, software de geometria dinâmica, vídeo sob demanda?

Questão aberta relacionada às TED de maneira geral

Você deseja fazer algum comentário adicional?

Fonte: Elaborado pelos autores (2020).

Para esta pesquisa, foram consideradas apenas 5 (cinco) TED (fórum de discussão, planilha eletrônica, sistema de gestão de aprendizagem, software de geometria dinâmica e vídeo sob demanda) entre as 12 (doze) identificadas no mapeamento sistemático mencionado na seção 2 deste artigo. Optou-se por escolher essas TED porque foram as tecnologias mais utilizadas (maior número de artigos relacionados) no referido mapeamento.

\section{Resultados e discussões}

Conforme descrito na seção anterior, dezessete (17) professores participaram de uma pesquisa de opinião, da qual foram coletados dados por meio da aplicação de um questionário fundamentado no Modelo TAM. A seguir, serão apresentados os resultados e discussões desses dados.

\subsection{Análise quantitativa dos dados}

Nesta seção, discute-se a aceitação das TED em três tópicos, os quais abordam, respectivamente, a facilidade de uso percebida, a utilidade percebida e a intenção de uso dos participantes da pesquisa. O Quadro contendo as frequências de respostas relativas a cada uma das TED, pode ser visualizado no seguinte endereço: http://bit.ly/AceitacaoTED.

\subsubsection{Facilidade de uso percebida}

Em relação ao construto facilidade de uso percebida (Figura 1), foi aferido o quanto o professor acha fácil usar as TED. Analisando individualmente cada tecnologia, observouse, baseado na apresentação geral dos dados, que vídeo sob demanda (81\%), seguido de fórum de discussão (79\%) e software de geometria dinâmica (79\%) foram as TED consideradas 
mais fáceis de usar. A planilha eletrônica (68\%) e o sistema de gestão de aprendizagem (59\%) apresentaram os menores índices de facilidade de uso percebida.

Figura 1. Facilidade de uso percebida - apresentação dos dados.

\begin{tabular}{|c|c|c|c|c|c|c|c|c|c|c|c|c|c|}
\hline \multirow[b]{2}{*}{ TED } & \multicolumn{10}{|c|}{ Distribuição de frequencia das respostas* } & \multirow{2}{*}{$\begin{array}{l}\text { Percentual de respostas } \\
\text { negativas ou neutras } \\
100 \%\end{array}$} & \multirow{2}{*}{\multicolumn{2}{|c|}{ 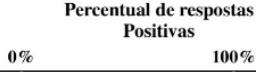 }} \\
\hline & $\mathbf{N}$ & $\%$ & $\mathbf{N}$ & $2 \%$ & $\mathbf{N}$ & $3 \%$ & $\mathbf{N}$ & $\%$ & $\mathbf{N}$ & $\%$ & & & \\
\hline Fórum de discussão & 5 & $7 \%$ & 4 & $6 \%$ & 5 & $7 \%$ & 37 & $54 \%$ & 17 & $25 \%$ & $21 \%$ & & $79 \%$ \\
\hline Planilha eletrônica & 5 & $7 \%$ & 11 & $16 \%$ & 6 & $9 \%$ & 36 & $53 \%$ & 10 & $15 \%$ & $32 \%$ & & $68 \%$ \\
\hline Sistema de Gestão de aprendizagem & 5 & $7 \%$ & 12 & $18 \%$ & 11 & $16 \%$ & 33 & $49 \%$ & 7 & $10 \%$ & $41 \%$ & & $59 \%$ \\
\hline Software de geometria dinâmica & 2 & $3 \%$ & 8 & $12 \%$ & 4 & $6 \%$ & 37 & $54 \%$ & 17 & $25 \%$ & $21 \%$ & & $79 \%$ \\
\hline Vídeo sob demanda & 2 & $3 \%$ & 7 & $10 \%$ & 4 & $6 \%$ & 37 & $54 \%$ & 18 & $26 \%$ & $19 \%$ & & $81 \%$ \\
\hline
\end{tabular}

Fonte: Elaborado pelos autores (2020).

Ao analisar as declarações de forma detalhada, observou-se que as declarações [D01] - "As tecnologias são fáceis de serem manipuladas" e [D04] - "O uso das tecnologias facilita a minha explicação dos conteúdos abordados em sala de aula" obtiveram os maiores percentuais positivos em todas as TED analisadas. Sendo a [D01] unânime na percepção dos professores ao avaliarem a TED fórum de discussão.

Observou-se que as declarações [D02] - "Estou acostumado a elaborar aulas com o emprego de tecnologias" e [D03] - "Consigo utilizar as tecnologias sem a necessidade de um curso de formação" contribuíram negativamente para o resultado da TED sistema de gestão de aprendizagem, pois apresentaram os maiores percentuais negativos ou neutros.

\subsubsection{Utilidade percebida}

No construto utilidade percebida (Figura 2), foi medido o quanto o professor admite que o emprego das TED poderá contribuir na prática, ou seja, se as ferramentas tecnológicas são capazes de melhorar o ensino-aprendizagem de matemática. O software de geometria dinâmica foi a TED que apresentou o maior índice de repostas positivas $(93 \%)$, logo depois vídeo sob demanda (82\%), fórum de discussão (72\%), planilha eletrônica (65\%) e o sistema de gestão de aprendizagem (57\%).

Figura 2. Utilidade percebida - apresentação dos dados.

\begin{tabular}{|c|c|c|c|c|c|c|c|c|c|c|c|c|c|}
\hline \multirow[b]{2}{*}{ TED } & \multicolumn{10}{|c|}{ Distribuição de frequencia das respostas* } & \multirow{2}{*}{$\begin{array}{l}\text { Percentual de respostas } \\
\text { negativas ou neutras } \\
100 \%\end{array}$} & \multirow{2}{*}{\multicolumn{2}{|c|}{$\begin{array}{cc} & \begin{array}{c}\text { Percentual de respostas } \\
\text { Positivas } \\
0 \%\end{array} \\
\end{array}$}} \\
\hline & $\mathrm{N}$ & $\%$ & $\mathbf{N}$ & $2 \%$ & $\mathbf{N}$ & $3 \%$ & $\mathbf{N}$ & $\%$ & $\mathrm{~N}$ & $5 \%$ & & & \\
\hline Fórum de discussão & 2 & $3 \%$ & 7 & $10 \%$ & 10 & $15 \%$ & 39 & $57 \%$ & 10 & $15 \%$ & $28 \%$ & & $72 \%$ \\
\hline Planilha eletrônica & 1 & $1 \%$ & 8 & $12 \%$ & 15 & $22 \%$ & 33 & $49 \%$ & 11 & $16 \%$ & $35 \%$ & & $65 \%$ \\
\hline Sistema de Gestão de aprendizagem & 1 & $1 \%$ & 7 & $10 \%$ & 21 & $31 \%$ & 25 & $37 \%$ & 142 & $21 \%$ & $43 \%$ & & $57 \%$ \\
\hline Software de geometria dinâmica & 1 & $1 \%$ & 2 & $3 \%$ & 2 & $3 \%$ & 37 & $54 \%$ & 26 & $38 \%$ & $7 \%$ & & $93 \%$ \\
\hline Vídeo sob demanda & 2 & $3 \%$ & 6 & $9 \%$ & 4 & $6 \%$ & 34 & $50 \%$ & 22 & $32 \%$ & $18 \%$ & & $82 \%$ \\
\hline
\end{tabular}

Fonte: Elaborado pelos autores (2020).

Quando questionados acerca da TED software de geometria dinâmica, constatou-se que todos $(100 \%)$ os professores manifestaram respostas positivas na declaração [D08] "Auxiliam no ensino-aprendizagem de matemática". Ainda sobre a análise dessa tecnologia, observou-se que as demais declarações [D07] - "O uso das TED permite uma maior visualização e compreensão dos conteúdos ministrados", [D05] - "Proporcionam aulas mais dinâmicas e atrativas" e [D06] - "Motivam os alunos a serem mais participativos nas aulas" tiveram elevados percentuais de aprovação, com índices iguais ou superiores a $88 \%$ em cada uma delas. 
Notou-se que, nas outras TED, a declaração [D06] - "Motivam os alunos a serem mais participativos nas aulas" apresentou os maiores percentuais negativos ou neutros, com índices entre $35 \%$ e $47 \%$.

\subsubsection{Intenção de uso}

Quanto ao construto intenção de uso, buscou-se identificar, nos participantes da pesquisa, se existe interesse em utilizar as TED. Conforme ilustrada na Figura 3, a intenção de uso dos professores foi distribuída da seguinte forma: software de geometria dinâmica (91\%), vídeo sob demanda (84\%), fórum de discussão (81\%), planilha eletrônica (79\%) e o sistema de gestão de aprendizagem (78\%). Este construto alcançou, ao analisar individualmente as TED, os maiores percentuais de respostas positivas quando comparadas aos outros construtos (facilidade de uso percebida e utilidade percebida), à exceção do resultado obtido pela tecnologia software de geometria dinâmica quando avaliada no construto utilidade percebida $(93 \%)$.

Figura 3. Intenção de uso - apresentação dos dados.

\begin{tabular}{|c|c|c|c|c|c|c|c|c|c|c|c|c|}
\hline \multirow{2}{*}{ TED } & \multicolumn{10}{|c|}{ Distribuição de frequencia das respostas* } & \multirow{2}{*}{$\begin{array}{l}\text { Percentual de respostas } \\
\text { negativas ou neutras } \\
100 \%\end{array}$} & \multirow{2}{*}{$\begin{array}{l}\text { Percentual de respostas } \\
\text { Positivas } \\
\\
100 \%\end{array}$} \\
\hline & $\mathbf{N}$ & $\%$ & $\mathrm{~N}$ & $2 \%$ & $\mathrm{~N}$ & ${ }^{3} \%$ & $\mathbf{N}$ & $\%$ & $\mathrm{~N}$ & $\%$ & & \\
\hline Fórum de discussão & 1 & $1 \%$ & 8 & $12 \%$ & 4 & $6 \%$ & 35 & $51 \%$ & 20 & $29 \%$ & $19 \%$ & $81 \%$ \\
\hline Planilha eletrônica & 1 & $1 \%$ & 6 & $9 \%$ & 7 & $10 \%$ & 33 & $49 \%$ & 21 & $31 \%$ & $21 \%$ & $79 \%$ \\
\hline Sistema de Gestão de aprendizagem & 0 & $0 \%$ & 6 & $9 \%$ & 9 & $13 \%$ & 28 & $41 \%$ & 25 & $37 \%$ & $22 \%$ & $78 \%$ \\
\hline Software de geometria dinâmica & 0 & $0 \%$ & 3 & $4 \%$ & 3 & $4 \%$ & 30 & $44 \%$ & 32 & $47 \%$ & $9 \%$ & $91 \%$ \\
\hline Vídeo sob demanda & 1 & $1 \%$ & 5 & $7 \%$ & 5 & $7 \%$ & 33 & $49 \%$ & 24 & $35 \%$ & $16 \%$ & $84 \%$ \\
\hline
\end{tabular}

Observando individualmente as declarações, constatou-se que [D10] - "Recomendo o emprego de TED em sala de aula" obteve os maiores percentuais positivos (94\%) quando questionados sobre as TED fórum de discussão, software de geometra dinâmica e vídeo sob demanda.

Ao analisar o percentual de respostas positivas relacionado à declaração [D11] "Gostaria de realizar capacitação em TED apoiadas ao ensino-aprendizagem de matemática" em cada uma das cinco TED, observou-se uma aceitação com percentuais entre $76 \%$ e $82 \%$. O índice positivo é ainda maior quando analisada a declaração [D12] - "Estou motivado(a) a utilizar as TED nas disciplinas que leciono", com valores entre 82 e $94 \%$, sendo quase unânime (94\%) quando avaliado o software de geometria dinâmica isoladamente. Levando em conta os resultados obtidos, evidencia-se interesse dos professores em usar as TED, já que há motivação para utilizá-las e entusiasmo em realizar capacitação.

\subsection{Análise qualitativa dos dados}

Nesta seção, são apresentados os pontos positivos e negativos capazes de afetar a adoção das TED avaliadas, segundo a opinião dos professores participantes da pesquisa. Os dados coletados foram categorizados conforme a Figura 4.

$\mathrm{Na}$ avaliação dos professores, o número de registros de pontos positivos foi superior ao de pontos negativos. O software de geometria dinâmica foi a TED que apresentou o maior número de observações positivas.

Ao analisar individualmente as variáveis externas de cada TED, com base na Figura 4, constatou-se que as variáveis interação, mediação, visualização e aprendizagem foram registradas pelos professores em todas as TED, exceto na tecnologia vídeo sob demanda, em que foram apontadas apenas as variáveis visualização e aprendizagem.

Em relação à infraestrutura dos Campi e às condições de trabalho proporcionadas aos professores, o código [N03] - "Alguns alunos não têm as ferramentas tecnológicas V. $19 \mathrm{~N}^{\mathrm{o}} 1$, julho, 2021 DOI: https://doi.org/10.22456/1679-1916.118393 RENOTE 
necessárias para utilização dessa TED" foi apontado em todas as TED. Ainda sobre a infraestrutura dos Campi, o código [N04] - "A instituição nem sempre dispõe de infraestrutura devida (computador/aluno e laboratórios ocupados)" foi pontuado nas seguintes TED: planilha eletrônica e software de geometria dinâmica. Percebe-se que a falta de ferramentas tecnológicas ou a indisponibilidade desses recursos pelos alunos ainda são desafios que precisam ser superados pela instituição.

Figura 4. Fontes de pontos positivos e negativos identificados.

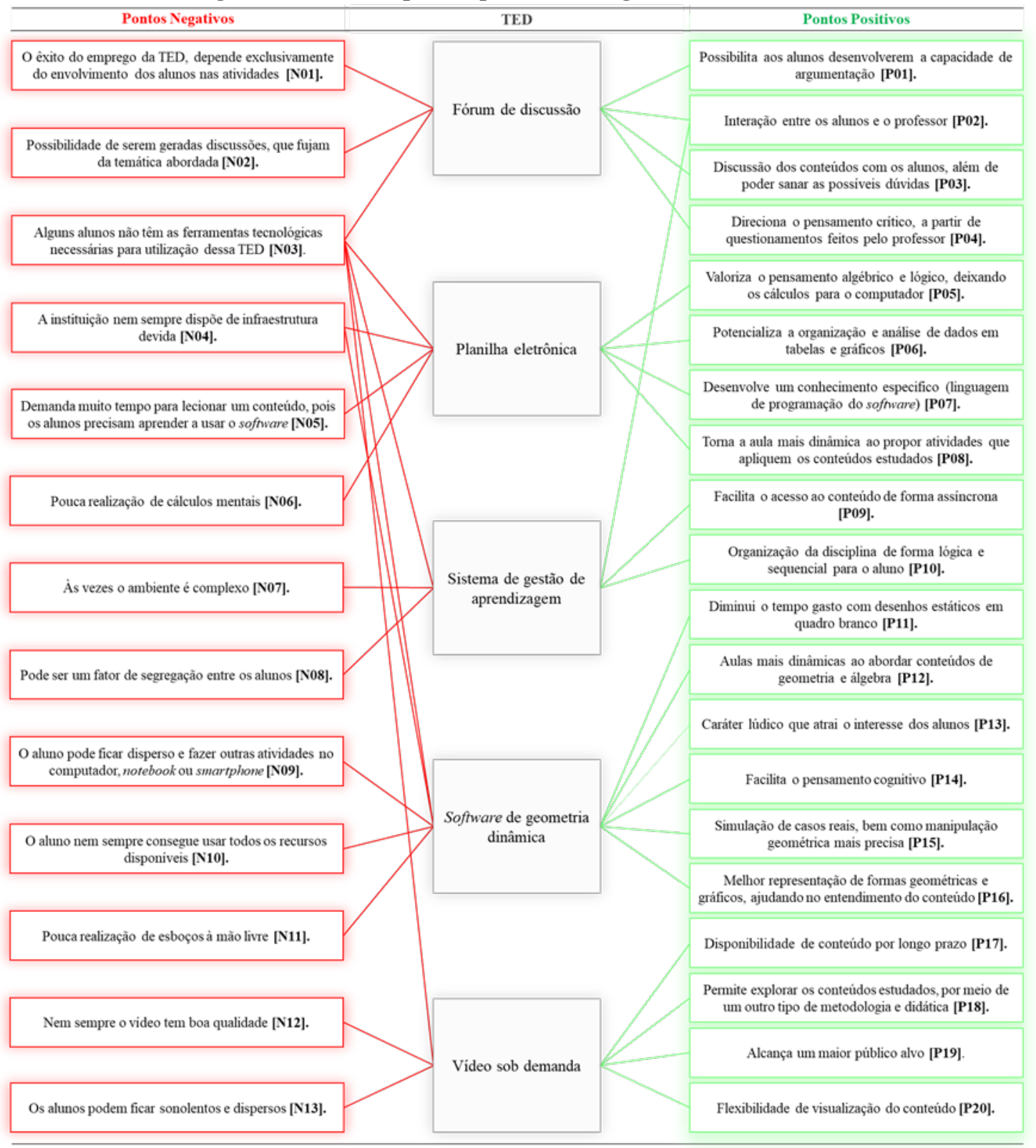

Fonte: Elaborado pelos autores (2020).

Em relação à interface, constatou-se que as TED possuem uma área de interação atraente, permitindo um ambiente satisfatório de mediação, comunicação, visualização e aprendizagem. Chegou-se a esse entendimento devido às avaliações positivas que convergiram para os códigos: [P02] - "Interação entre os alunos e o professor"; [P03] "Discussão dos conteúdos com os alunos, além de poder sanar as possíveis dúvidas"; [P04] - "Direciona o pensamento crítico, a partir de questionamentos feitos pelo professor"; [P06] - "Potencializa a organização e análise de dados em tabelas e gráficos"; [P07] - "Desenvolve um conhecimento especifico (linguagem de programação do software)"; [P08] - "Torna a aula mais dinâmica ao propor atividades que apliquem os conteúdos estudados"; [P09] V. $19 \mathrm{~N}^{\mathrm{o}} 1$, julho, 2021 
"Facilita o acesso ao conteúdo de forma assíncrona"; [P10] - "Organização da disciplina de forma lógica e sequencial para o aluno"; [P12] - "Aulas mais dinâmicas ao abordar conteúdos de geometria e álgebra"; [P13] - "Caráter lúdico que atrai o interesse dos alunos; [P14] "Facilita o pensamento cognitivo"; [P15] - "Simulação de casos reais, bem como manipulação geométrica mais precisa"; [P16] - "Melhor representação de formas geométricas e gráficos, ajudando no entendimento do conteúdo"; [P18] - "Permite explorar os conteúdos estudados, por meio de um outro tipo de metodologia e didática"; [P20] "Flexibilidade de visualização do conteúdo".

Embora haja semelhanças na percepção dos professores ao analisarem as variáveis externas, percebeu-se haver particularidades em cada uma das TED. Na tecnologia fórum de discussão, foi assinalado como um dos pontos negativos o código [N01] - "O êxito do emprego da TED depende exclusivamente do envolvimento dos alunos nas atividades", enquanto na TED planilha eletrônica, os aspectos negativos indicados foram os códigos [N05] - "Demanda muito tempo para lecionar um conteúdo, pois os alunos precisam aprender a usar o software" e o [N06] - "Pouca realização de cálculos mentais". Já em relação à TED sistema de gestão de aprendizagem, foi exposto o código [N07] - "Às vezes o ambiente é complexo", enquanto na TED software de geometria dinâmica os códigos [N10] - "Os alunos nem sempre conseguem usar todos os recursos disponíveis" e [N11] - "Pouca realização de esboços à mão livre". Por fim, na TED vídeo sob demanda foram apontados como pontos negativos os códigos [N12] - "Nem sempre o vídeo tem boa qualidade" e [N13] - "Os alunos podem ficar sonolentos e dispersos".

Diante dos pontos positivos e negativos identificados pelos professores (Figura 4), percebe-se que a maioria deles conseguem identificar as ferramentas necessárias para preparar uma aula, qual o melhor conteúdo a ser explorado e o que pretendem alcançar com os recursos utilizados.

\section{Considerações finais}

Este trabalho corresponde à segunda etapa de uma pesquisa mais ampla, que teve o propósito de auxiliar o planejamento da formação de professores de matemática em uma Instituição de Ensino Médio do Brasil. Na primeira etapa da referida pesquisa, foi conduzido um mapeamento sistemático, o qual permitiu identificar Tecnologias Educacionais Digitais que, tipicamente, são utilizadas no contexto brasileiro (DE VASCONCELOS et al., 2020). Neste artigo, especificamente, o objetivo foi avaliar a aceitação dos professores do IFSertãoPE em relação à utilização das TED adotadas para o ensino-aprendizagem de Matemática.

Os resultados apontam que o software de geometria dinâmica se destacou como a TED melhor avaliada, tanto na abordagem quantitativa (nos construtos utilidade percebida e intenção de uso do Modelo de Aceitação de Tecnologia) como na qualitativa. Além dessa TED, outras também obtiveram elevado percentual de respostas positivas: fórum de discussão e vídeo sob demanda. Sugere-se que essas duas últimas TED sejam empregadas de forma associada aos softwares de geometria dinâmica, ampliando, assim, as possibilidades de estratégias para o ensino-aprendizagem de matemática.

Acredita-se que este artigo possa auxiliar no planejamento da formação de professores, não apenas pela indicação das TED, mas, principalmente, por realçar a percepção dos professores a respeito de aspectos que influenciam a aceitação dessas tecnologias nos processos de ensino e aprendizagem da Matemática.

Em relação às dificuldades encontradas para a realização deste artigo, destaca-se o fato de o estudo ter ocorrido durante o período de isolamento social, o que levou os pesquisadores, por consequência, a manter contato/interações com os participantes, exclusivamente, por meio virtual.

Quanto às limitações do trabalho, observa-se que a pesquisa envolveu a percepção de apenas um grupo reduzido de professores do IFSertãoPE. Sugere-se, então, a condução de V. $19 \mathrm{~N}^{\circ} 1$, julho, 2021 DOI: https://doi.org/10.22456/1679-1916.118393 RENOTE 
estudos com um público mais amplo, envolvendo a participação de docentes de outras instituições. Outra sugestão de pesquisa é que seja avaliada a aceitação dos estudantes em relação à utilização das TED adotadas para o ensino-aprendizagem de Matemática.

\section{Referências}

BASSO, Marcus; NOTARE, Márcia Rodrigues. Pensar-com tecnologias digitais de matemática dinâmica. RENOTE-Revista Novas Tecnologias na Educação, v. 13, n. 2, 2015. DOI: https://doi.org/10.22456/1679-1916.61432.

DA SILVA, Lucas T.; DA SILVA, Karina N.; GROENWALD, Claudia LO. A utilização de dispositivos móveis na educação matemática. Educação Matemática em Revista. Brasília, ano, v. 23, p. 59-76, 2018.

DAVIS, Dan et al. Encouraging Metacognition \& Self-Regulation in MOOCs through Increased Learner Feedback. In: Proceedings of the 6nd international conference on Learning Analytics and Knowledge, 2016. p. 17-22.

DE VASCONCELOS, Gibran Medeiros Chaves et al. Mapeamento das Tecnologias Educacionais Digitais adotadas para o ensino-aprendizagem de Matemática em instituições de Ensino Médio no Brasil. RENOTE, v. 18, n. 1, 2020. DOI: https://doi.org/10.22456/16791916.106045.

DIAS, Guilherme Ataíde et al. Technology Acceptance Model (TAM): avaliando a aceitaçao tecnológica do open journal systems (OJS). Informação \& Sociedade, v. 21, n. 2, 2011.

GRINSPUN, Mirian Paura Sabrosa Zippin; MANESCHY, Patricia; MOTA, Fernando. Desafios e perspectivas para juventude em um mundo de tecnologia challenges and prospects for youth in a world of technology. Tecnologia Educacional, p. 61-70, 2016.

HAIR, Joseph F. et al. Análise multivariada de dados. Bookman Editora, 2009.

LOPES, Thiago Beirigo; COSTA, Ademir Brandão da; OLIVEIRA, Ritianne de Fátima Silva de. Estudo de função afim utilizando o software Geogebra como ferramenta interativa. Revista Tecnologias na Educação, v. 8, n. 17, p. 1-16, 2016.

PERSICO, Donatella; MANCA, Stefania; POZZI, Francesca. Adapting the technology acceptance model to evaluate the innovative potential of e-learning systems. Computers in Human Behavior, v. 30, p. 614-622, 2014. DOI: https://doi.org/10.1016/j.chb.2013.07.045. PRODANOV, Cleber Cristiano; DE FREITAS, Ernani Cesar. Metodologia do trabalho científico: métodos e técnicas da pesquisa e do trabalho acadêmico-2 ${ }^{a}$ Edição. Editora Feevale, 2013.

RETZLAFF, Eliani et al. Ambiente Dinâmico Scratch no Ensino da Matemática. Tecné Episteme y Didaxis TED, p. 1-10, 2018.

VIEIRA, Sonia. Como elaborar questionários. São Paulo: Atlas, 2009. 